\title{
DyNAMic Modelling OF MOBILE Robots BASED ON ITS 3D CAD MODEL
}

\author{
Vjekoslav Damic, Maida Cohodar \& Munir Muratovic
}
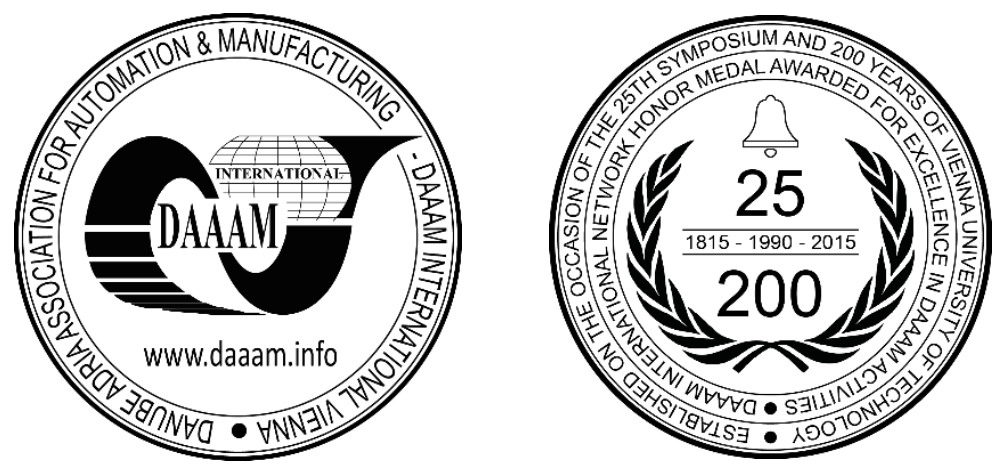

This Publication has to be referred as: Damic, V[jekoslav]; Cohodar, M[aida] \& Muratovic, M[unir] (2017). Dynamic Modelling of Mobile Robots Based on its 3D CAD Model, Proceedings of the 28th DAAAM International Symposium, pp.0144-0149, B. Katalinic (Ed.), Published by DAAAM International, ISBN 978-3-902734-11-2, ISSN 1726-9679, Vienna, Austria

DOI: $10.2507 / 28$ th.daaam.proceedings.019

\begin{abstract}
Usage of the mobile robots is extended in all areas of human life and work. The investigation of their behaviour becomes popular among the researchers around the world. Dynamic modelling of the mobile robot can be simplified by using their 3D CAD model. In this paper, the dynamic model of mobile robot is developed by MATLAB/Simulink using previously created 3D CAD model in SOLIDWORKS. As example, the mobile robot Robotino by FESTO is chosen. Assembly of mobile robot in form of $\mathrm{xml}$ file is imported from SOLIDWORKS to MATLAB. Such approach provides automatically generation of dynamics of robot's mechanical subsystem. Then, the model can be modified and upgraded, for instance to organize it on several levels, to actuate the wheels by motors, etc. Validation of the dynamic model is performed by simulation.
\end{abstract}

Keywords: mobile robot; dynamic modelling; visualization; 3D CAD model

\section{Introduction}

Investigations of the robots attract attention of many researches around the world in different areas. Modelling and simulation are recognized as powerful tool for solving many problems in robotics. Analysis of industrial robots using bond graphs by visualization is presented in [2], where BondSim is used for dynamic analysis of robot and BondSim3D Visual application is used for its visualization. Simulation of hobby robot by MATLAB is subject of analysis in [4]. Also, the huge research area is modelling of mobile robotics behaviours. The kinematics and dynamic analysis of mobile robot was done in [5]. Investigation of behaviour of group of mobile robots is subject of research in [8,9]. A presentation of working scenarios and mobile robot working algorithms in Bionic Assembly System and their realization in AnyLogic software is given in [10].

The paper proposes procedure for the dynamic analysis of mobile robot by MATLAB/Simulink based on previously created 3D CAD model [11]. Proposed approach is explained on example of mobile robot Robotino from FESTO [6]. Robotino is subject of research of [1], where it is used for arrangement of remote education. Modelling of Robotino by bond graphs is presented in [3].

In this research, the dynamic model of Robotino is automatically developed by importing its 3D CAD model from SOLIDWORKS to MATLAB following the idea from [7]. Dimensions of robot parts are roughly measured and estimated. 
The obtained robot's dynamic model is reorganized systematically into more hierarchical levels and its validation was conducted by simulation. It can be upgraded and used to test different control algorithms, trajectory planning, collision avoidance, etc.

\section{3D CAD model of Robotino}

Mobile robot Robotino (Fig.1a) is equipped by three omnidirectional wheels. They are powered by dc motors, which are denoted by manufacturer FESTO as motor 1 (M1), motor 2 (M2) and motor 3 (M3). Wheels and corresponding motors are denoted inFig. 1 by red, green and blue colour, respectively. Each wheel is composed of the roller supporter and six rollers, three rollers per each side, shifted by $120^{\circ}$ with respect to each other. Rollers can rotate about their axes.

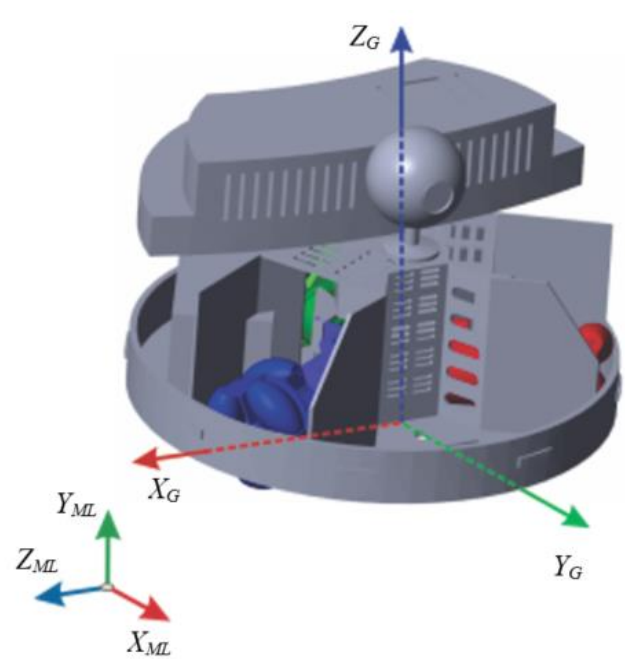

a)

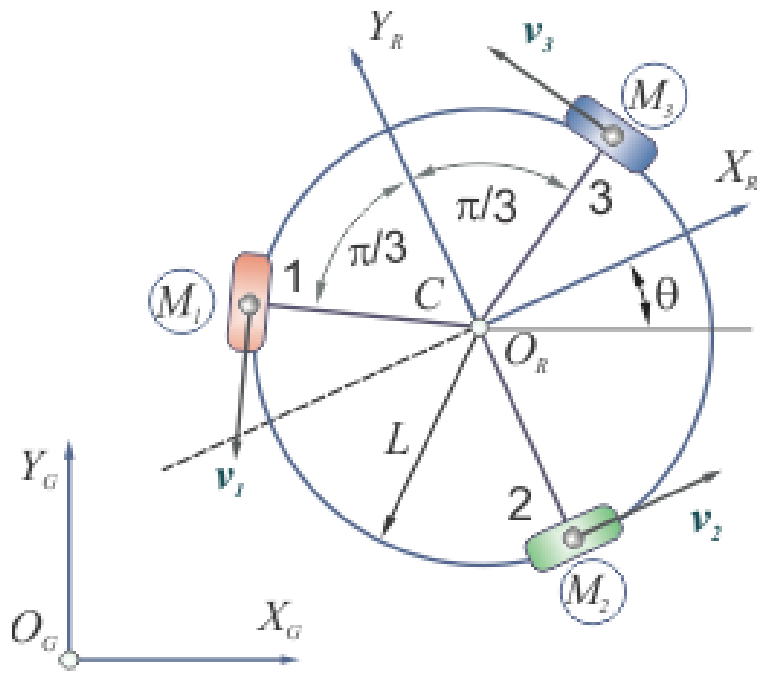

b)

Fig. 1. Robotino with assigned coordinate frames: a) the global frame; b) the local frame

As previously emphasized, the first step to develop the mobile robot dynamic model is creating of their 3D CAD model. Using SOLIDWORKS 3D CAD models of the robot's parts are generated (Fig.2). For simplicity unnecessary details are omitted, without loss of robot functionality. 3D CAD model of robot is taken from [11].

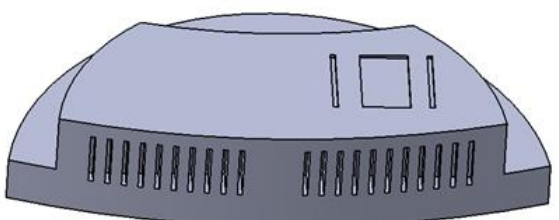

cover

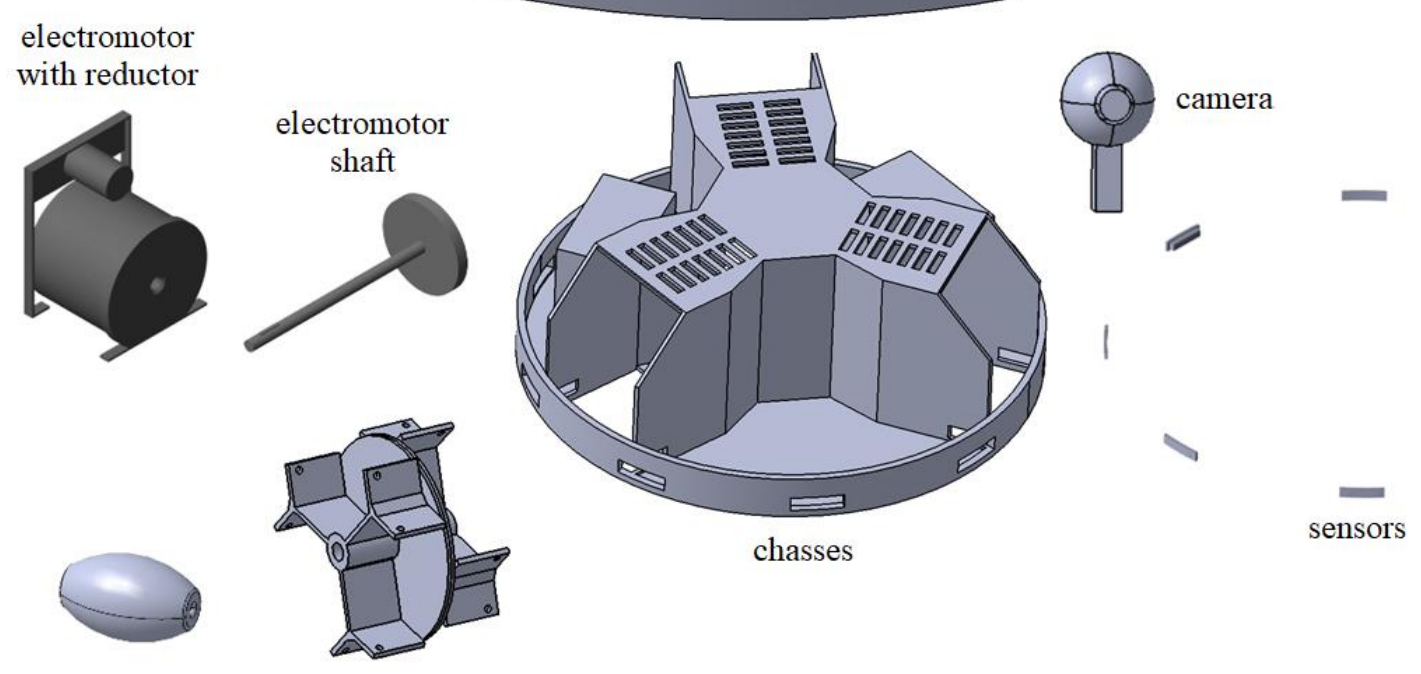

roller roller supporter

Fig. 2. Robotino parts 
Robot's parts are combined in the assembly by exactly defined mating between them using the SOLIDWORKS instruction. Finally, the assembly shown in Fig.1a has been imported using SimMechanic link (ver. 4.7 of Matlab 2015b and Windows x64) in the form of xml file, and their parts as stl files.

\section{Dynamic model}

By importing the robot assembly into MATLAB in form of xlm file, that is obtained using SOLIDWORKS, the dynamic model of the Robotino has been automatically developed. It is realized as single level structure. Taking into account that robot consists of many parts the model is pretty unclear and complicated. Hence, it can be organized systematically into more levels by putting group of parts into subsystems, as depicted in Figs. 3 and 4.

There is several blocks only in the first level: the block which represents the dynamic model of Robotino, planar joint, two sensors to measure robot positons and blocks to define world coordinate frame, appropriate solver and the mechanical simulation environment for the robot (for instance gravity, constraint solver type, tolerances, linearization, etc.). It is important to emphasize that the coordinate system in which the robotic assembly is made, corresponds to the world coordinate system in MATLAB, $O_{M L} X_{M L} Y_{M L} Z_{M L}$.

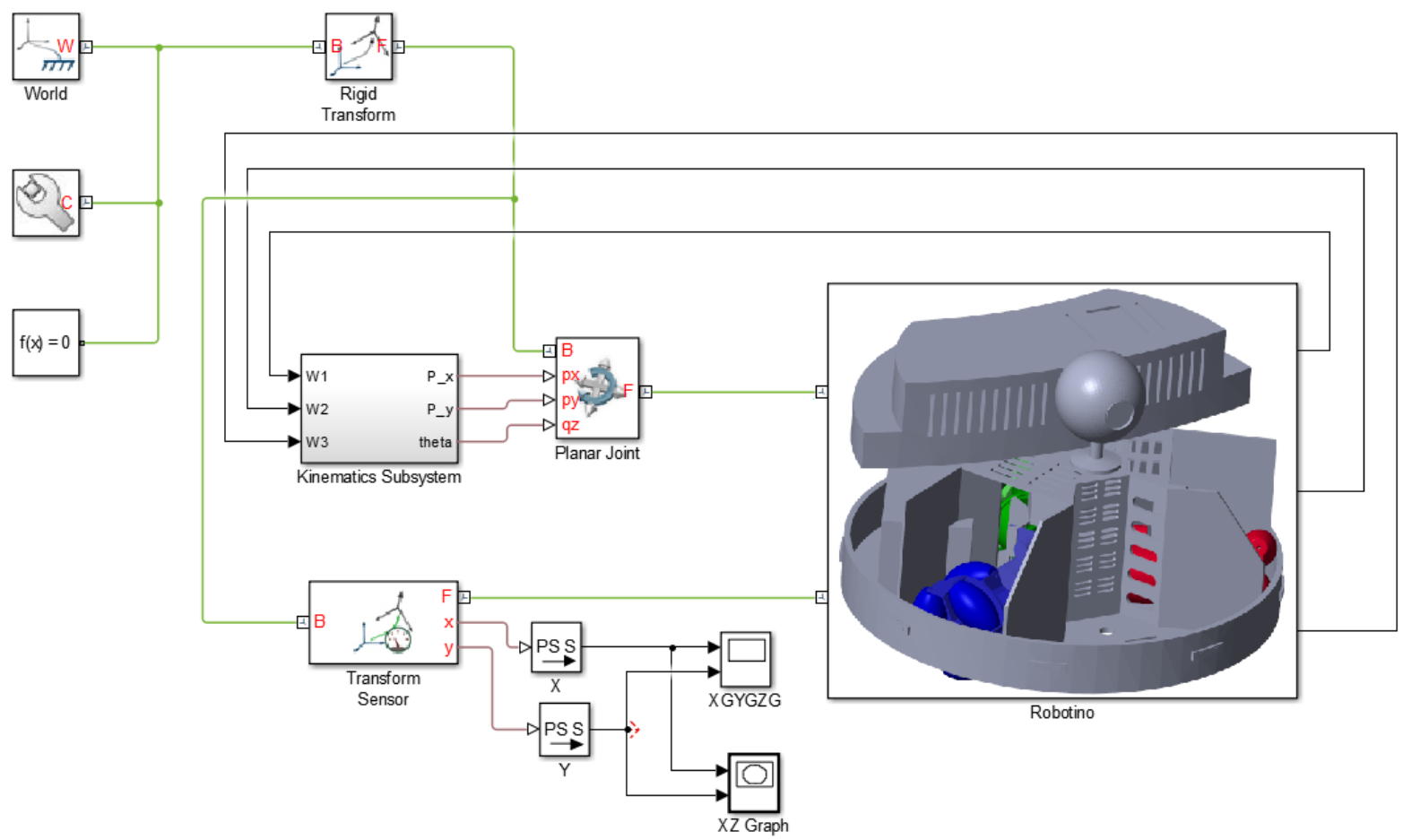

Fig. 3. The system level dynamic model of Robotino,

Due to fact that Robotino manufacturer in the robot manuals and other documentations uses the global coordinate frame $O_{G} X_{G} Y_{G} Z_{G}$ (as shown in Fig.1a) all quantities should be transform to $O_{M L} X_{M L} Y_{M L} Z_{M L}$ using block 'Rigid transform', as depicted on the top of Fig.3. This transformation is given by:

$$
\mathbf{R}_{M L}^{G}=\left[\begin{array}{lll}
0 & 0 & 1 \\
1 & 0 & 0 \\
0 & 1 & 0
\end{array}\right] .
$$

Following idea from [7], the robot is connecting to the ground with the planar joint providing two translator motions $p_{x}$ and $p_{y}$ along $X_{G}$ and $Y_{G}$-axis and rotation $\theta$ about $Z_{G}$-axis. Inputs for the planar joint are given by:

$$
\begin{aligned}
& p_{x}=\int v_{x} d t=-\int v_{1} \cdot \cos \left(\theta+\frac{\pi}{3}\right) d t+\int v_{2} \cdot \cos (\theta) d t-\int v_{3} \cdot \cos \left(\theta-\frac{\pi}{3}\right) d t \\
& p_{y}=\int v_{y} d t=-\int v_{1} \cdot \sin \left(\theta+\frac{\pi}{3}\right) d t+\int v_{2} \cdot \sin (\theta) d t-\int v_{3} \cdot \sin \left(\theta-\frac{\pi}{3}\right) d t \\
& \theta=\int \frac{\left(\omega_{1}+\omega_{2}+\omega_{3}\right) \cdot R}{3 L} d t
\end{aligned}
$$

where $v_{i}$ and $\omega_{i}$ are linear and angular velocities of wheels $(i=1,2,3), R$ is wheel radius, $L$ is radius of mobile platform. 


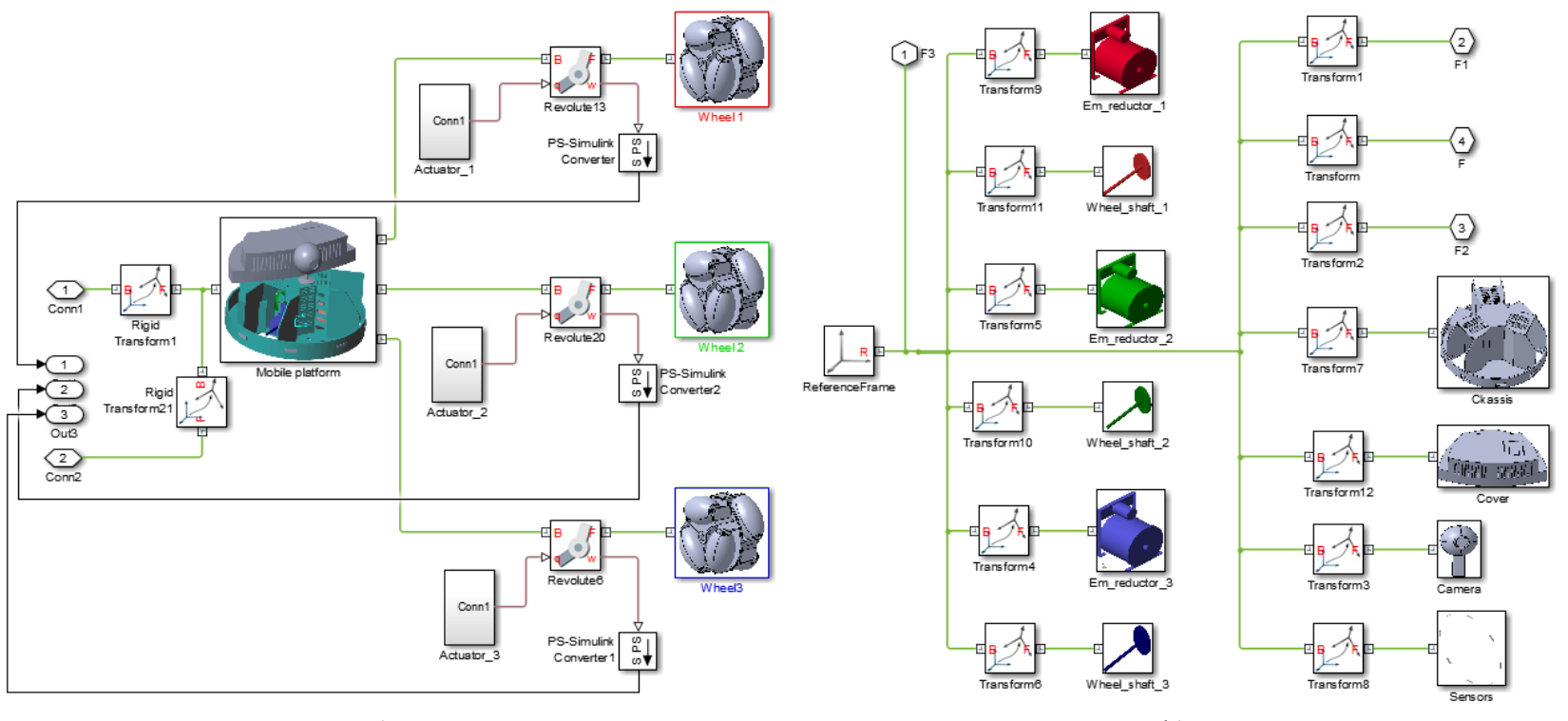

a)

b)

Fig. 4. The structure: a) 'Robotino' block; b) 'Mobile platform' block

Structure of Robotino on the next level of decomposition is shown in Fig.4a. The Mobile platform, three omnidirectional wheels, and their actuators, are the basic components presented on this level. Structure of 'Mobile platform' is depicted in Fig.4b. In order to taking acount mass of electromotors and reductors, they are presented as rigid bodies. Their funcionalities can be represented by Actuator_i $(i=1,2,3)$ blocks.

Strucuture of wheels are presented in Fig. 5a. Ahead each component there is block 'Rigid transform', which provides the appropriate coordinate transformations. Each component is realized as a rigid body (Fig.5b), whose mass and geometric properties are automatically calculated from SOLIDWORKS 3D CAD models.

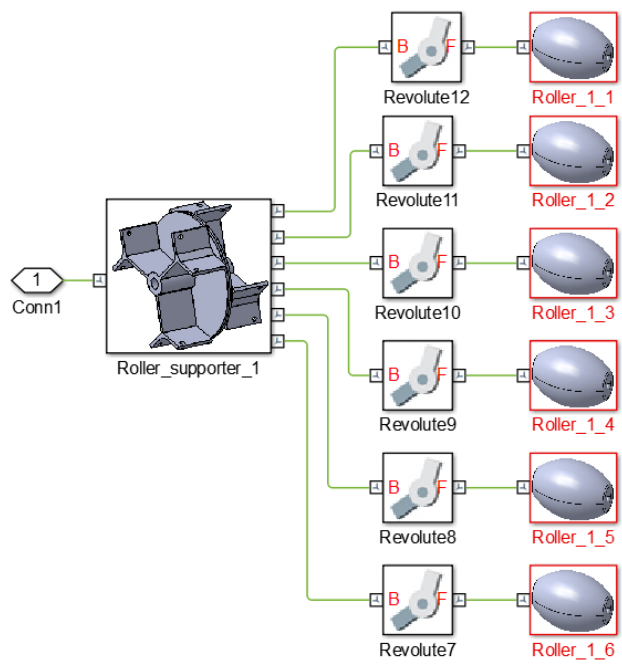

a)

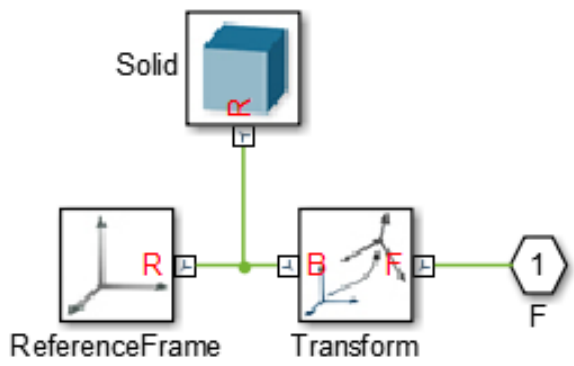

b)

Fig. 5. The structure of: a) 'Wheel' block; b) The rigid body block

\section{Simulation results}

To verify developed dynamic model of Robotino a simple experiment is performed. Wheels are powered in such a way that their angular accelerations change in the time as shown in Fig.6. Robot moves along $Y_{G}$ axis during the first $4 \mathrm{~s}$, because wheels 1 and 3 are actuated by the same angular velocities, but of the opposite sign. During this motion, the wheel 2 is not powered. In the next $5.1 \mathrm{~s}$ all three wheels rotate with the same angular velocity assuring that the robot just rotates about $Z_{G}$ axis, as shown in Fig.7. Finally, during $9.1 \mathrm{~s}$ to $15 \mathrm{~s}$ (the end of the simulation time) the robot moves along $Y_{G}$ axis, similarly to motion in the first phase (Fig.8). 


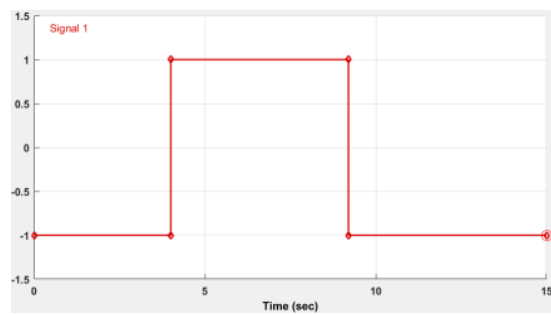

a)

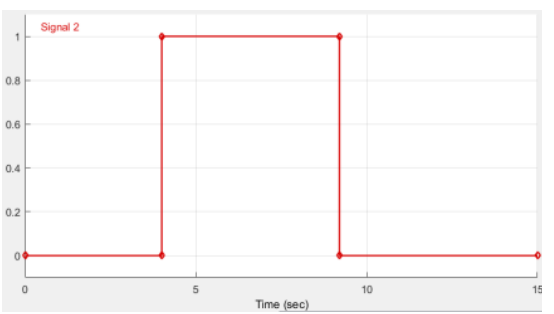

b)

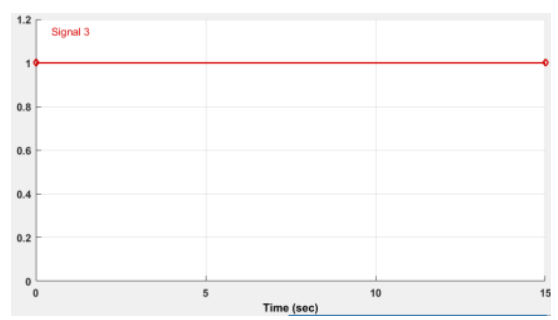

c)

Fig. 6. The angular acceleration of wheel: a) 1; b) 2; c) 3;
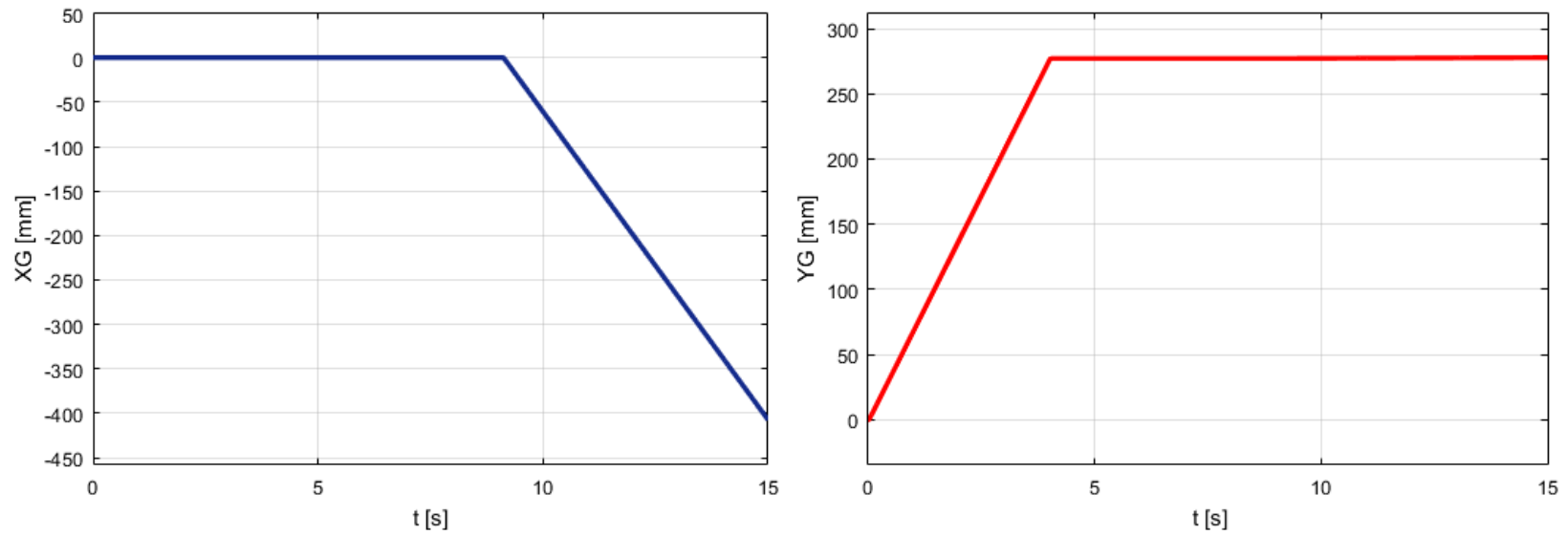

Fig. 7. $X$ and $Y$ coordinates of Robotino in $O_{G} X_{G} Y_{G} Z_{G}$

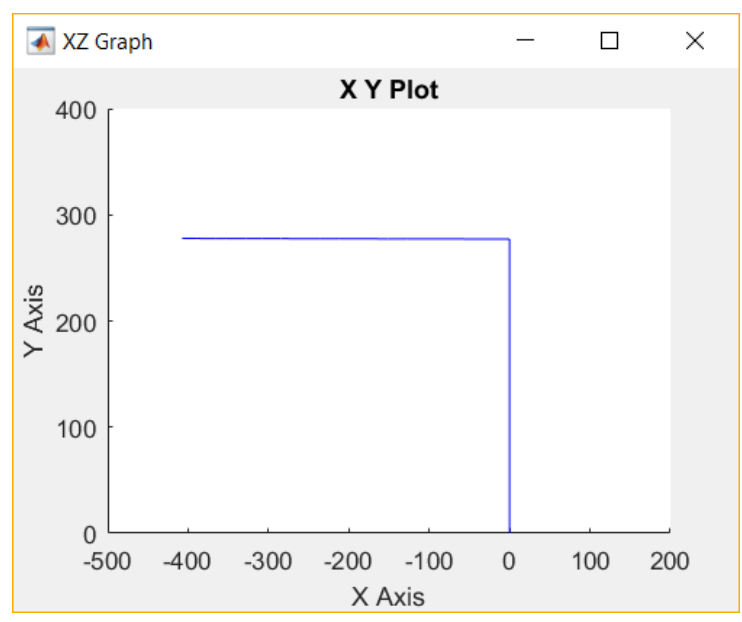

Fig. 8. The Robotino motion in plane in $O_{G} X_{G} Y_{G}$

\section{Conclusion}

The procedure for development of dynamic model of mobile robot based on its 3D CAD model is explained in the paper on example of mobile robot FESTO Robotino. Firstly, the 3D CAD model is developed using SOLIDWORKS and exported in form of $\mathrm{xml}$ file. The file is imported to MATLAB's SimMechanics module (MATLAB/Simulink ver. 2015b). The mass and geometric parameters including the all necessary transformations are automatically calculated from the 3D CAD model (assuming the material properties of the each robot part are defined previously). This approach provides a comfortable and simple way to develop the dynamic model of the mechanical part of the mobile robot.

The obtained dynamic model is realized as a single level (flat) structure. It could be changed by grouping some of the parts into the subsystems and thus providing systematically the model representation on more hierarchical levels. Model can be also upgraded by adding appropriate control structure, the wheel actuators, etc. assuring an excellent research platform for investigation of the robot behaviour. Comparison of different control algorithms, and path planning, work in unstructured environmental would be direction of future investigation. 


\section{References}

[1] Breido, I. V.; Markvardt, R. V. \& Zarnitcin, A.(2012) Modernization of communication interface between mobile robot Robotino and Matlab software, Annals of DAAAM for 2012 \& Proceedings of the 23rd International DAAAM Symposium, ISBN 978-3-901509-91-9, ISSN 2304-1382, pp 1107 - 1110, Editor B[ranko] Katalinic, Published by DAAAM International, Vienna, Austria

[2] Damic, V. \& Cohodar, M. (2017). Multibody System Modeling, Simulation, and 3D Visualization, Chapter 17, pp.627-671, in book (C) Springer International Publishing Switzerland 2017, W. Borutzky (ed.), Bond Graphs for Modelling, Control and Fault Diagnosis of Engineering Systems, DOI 10.1007/978-3-319-47434-2_17

[3] Damic, V.; Cohodar, M. \& Omerspahic, A. (2013). Dynamic Analysis of an Omni-directional Mobile Robot, Proceedings of 17th Onternational Research/Expert Conference TMT 2013, 10-11 September 2013, Istanbul, ISSN, ISBN, Katalinic, B. (Ed.), pp. 14-17, Publisher, City, DOI

[4] Damic, V.; Cohodar, M.; \& Tvrtkovic, M. (2016). Inverse Dynamic Analysis of Hobby Robot uArm by Matlab/Simulink, Proceedings of the 27th DAAAM International Symposium, pp.0095-0101, B. Katalinic (Ed.), Published by DAAAM International, ISBN 978-3-902734-08-2, ISSN 1726-9679, Vienna, Austria DOI: 10.2507/27th.daaam.proceedings.014

[5] Du, Y. (2016). Kinematics and Dynamic Modeling and Simulation Analysis of Three-wheeled Mobile Robot, International Conference on Mechanics Design, Manufacturing and Automation (MDM 2016) ISBN: 978-1-60595354-0

[6] http://www.festo-didactic.com/ov3/media/customers/1100/544305_robotino_deen2.pdf, Accessed on: 2017-09-01

[7] https:/www.mathworks.com/videos/mobile-robot-simulation-for-collision-avoidance-with-simulink-90193.html, Accessed on: 2017-09-01

[8] Kirill, K. (2015). Software Architecture of Control System for Heterogeneous Group of Mobile Robos. Procedia Engineering, Vol.100, pp (278-282), doi: 10.1016/j.proeng.2015.01.368

[9] Klancar, G., Zupancic, B. \& Karba.R. (2007). Modelling and simulation of a group of mobile robots. Simulation Modelling Practice and Theory, 15:647-658.

[10] Kukushkin, I. K.; Katalinic, B.; Cesarec, P.; Zdyb, D. \& Kettler, R.. (2012) Modeling of mobile robot behavior for line-less Bionic Assembly System, Annals of DAAAM for 2012 \& Proceedings of the 23rd International DAAAM Symposium, ISBN 978-3-901509-91-9, ISSN 2304-1382, pp 0865 - 0870, Editor B[ranko] Katalinic, Published by DAAAM International, Vienna, Austria

[11] Muratovic, M. (2017). Modeling and simulation of mobile robot by Simulink, Faculty of Mechanical Engineering, University of Sarajevo, Master thesis 